\title{
The element abundance FIP effect in the quiet Sun ${ }^{\star}$
}

\begin{abstract}
P. R. Young
CCLRC Rutherford Appleton Laboratory, Chilton, Didcot, Oxfordshire, OX11 0QX, UK

e-mail: p.r.young@rl.ac.uk

Received 1 March 2005 / Accepted 2 May 2005

Abstract. The $\mathrm{Mg} / \mathrm{Ne}$ abundance ratio in the quiet Sun is measured in both network and supergranule cell centre regions through EUV spectra from the Coronal Diagnostic Spectrometer on SOHO. Twenty four sets of data over the period 1996 March to 1998 June (corresponding to solar minimum and the onset of the next cycle) are studied. Emission lines of the sequences $\mathrm{Ne}$ IV-VII and Mg V-VIII are simultaneously analysed by comparing with theoretical emissivities from the CHIANTI database to yield the $\mathrm{Mg} / \mathrm{Ne}$ abundance and emission measure over the temperature region $5.0 \leq \log T \leq 6.1$. The average enhancements over the photospheric $\mathrm{Mg} / \mathrm{Ne}$ abundance are found to be $1.25 \pm 0.10$ (network) and $1.66 \pm 0.23$ (cell centres), implying that the structures making up the transition region have close-to photospheric abundances. In particular, this implies that only a small fraction of the quiet Sun can connect to the solar wind where the $\mathrm{Mg} / \mathrm{Ne}$ abundance ratio is found to be factors $4-5$ greater than the photospheric value. The quiet Sun spectra are also utilised to determine the coronal density and temperature, leading to average values of $2.6_{-0.4}^{+0.5} \times 10^{8} \mathrm{~cm}^{-3}$ and $\log (T / \mathrm{K})=5.95 \pm 0.02$. No significant trend with the rise in solar activity during 1996-98 is found for any of the derived quantities, implying that quiet Sun regions show little dependence on the solar cycle.
\end{abstract}

Key words. Sun: abundances - Sun: transition region - Sun: UV radiation - Sun corona

\section{Introduction}

Abundance anomalies in the solar upper atmosphere and solar wind have been reported for over 40 years (see, e.g., review of Feldman \& Laming 2000), with the general result that elements with a low first ionization potential (FIP) are often enhanced over those with a high FIP. The separation between low and high FIP is around $10 \mathrm{eV}$ and thus, for example, $\mathrm{Fe}, \mathrm{Mg}$ and $\mathrm{Si}$ are considered low-FIP elements and $\mathrm{Ne}$ and $\mathrm{Ar}$ are considered high-FIP elements. This abundance anomaly pattern is generally referred to as the "FIP Effect". The FIP effect is important as it provides a constraint that models for the solar corona and solar wind must satisfy.

At solar minimum the slow solar wind originates from the quiet Sun and thus coronal and transition region plasma in the quiet Sun may be expected to show the FIP enhancement of a factor 4-5 typically found in the slow solar wind (Meyer 1985). Measurements of relative abundances are, however, rare for the quiet Sun partly due to limitations of solar instrumentation. The S082A spectroheliograph on Skylab had a slitless design that resulted in spectral and spatial dimensions being mixed and only small, isolated features could be studied for abundance work, ruling out quiet Sun observations on the solar disk. Feldman \& Widing (1993), however, were able to use the limbbrightening of transition region lines at the solar limb to isolate

$\star$ Table 2 is only available in electronic form at http://www.edpsciences.org emission from $\mathrm{Ne}$ VI and $\mathrm{Mg}$ VI ions, yielding a $\mathrm{Mg} / \mathrm{Ne}$ abundance enhancement over the photosphere of a factor 1.5-2.0.

Spadaro et al. (1996) used the average supergranule cell centre and network region spectra of Vernazza \& Reeves (1978) obtained with the S-055 instrument on board Skylab to investigate element abundances in the transition region and corona. They found no enhancement over photospheric values for the elements considered here (magnesium and neon), although there was only limited temperature overlap between the ions they considered.

A key advantage of the Normal Incidence Spectrometer of the Coronal Diagnostic Spectrometer on SOHO is that it obtains simultaneous spectral and spatial information for two large wavelength bands, allowing many ions from different species to be compared - an important requirement for abundance studies. There have been several analyses of CDS data that have yielded abundance measurements in quiet Sun regions. Landi \& Landini (1998) performed a differential emission measure (DEM) analysis on an active region and a nearby area of quiet Sun, yielding a $\mathrm{Mg} / \mathrm{Ne}$ enhancement over the photospheric value of 2.0 for the latter. MacPherson \& Jordan (1999) studied the intensities of helium lines in the quiet Sun and a by-product of their analysis was that the low-FIP enhancement over high-FIP elements was at most a factor 1.4, with a small variation between supergranule cell centres and network regions. Young \& Mason (1998) studied quiet Sun spectra averaged over many CDS rasters from 1996 and determined a $\mathrm{Mg} / \mathrm{Ne}$ ratio enhanced over the photospheric value 
by a factor 2 , with little difference between cell centres and network. It is to be noted that the present work differs from Young \& Mason (1998) through the analysis method used, and the separate study of individual data-sets over time.

All of the results from these works refer to the transition region. Results for the corona are not common in the literature. Laming et al. (1995) re-analysed an EUV rocket spectrum of Malinovsky \& Heroux (1973) to determine a FIP enhancement of 3-4. Above the solar limb, Feldman et al. (1998) have measured a low-FIP enhancement of a factor 4 using the SUMER instrument on board SOHO at a height of $1.03 R_{\odot}$. Another effect seen further off-limb above the quiet Sun is that of gravitational settling of heavier elements. Raymond et al. (1997) used SOHO/UVCS spectra obtained at $1.5 R_{\odot}$ to demonstrate that oxygen is underabundant relative to hydrogen by an order of magnitude; for ions of comparable mass, however, the FIP effect is still seen with a value around 4.

The present work uses spectra from CDS to determine $\mathrm{Mg} / \mathrm{Ne}$ relative abundance ratios in the quiet Sun for a number of data-sets over a period of 28 months from the most recent solar activity minimum (1996) through the rise in activity during 1997 and 1998. The aim is to provide definitive measurements for supergranule cell centres and network regions and to search for any long-term time variability.

\section{Observations}

The Coronal Diagnostic Spectrometer (CDS) is one of 12 scientific instruments on board the Solar and Heliospheric Observatory (SOHO) and obtains extreme ultraviolet (EUV) spectra over the range $150-800 \AA$ (Harrison et al. 1995). It consists of two separate spectrometers (the Grazing Incidence, GIS, and Normal Incidence, NIS) fed by the same telescope. Only data from the NIS are presented here. NIS simultaneously obtains spectral and one-dimensional spatial information in single exposures, with images of a region built up through rastering. Two wavebands are observed simultaneously: 308$381 \AA$ and 513-633 $\AA$. A rich selection of emission lines from different ion species are found in these bands, covering temperatures from $2 \times 10^{4}$ to $6 \times 10^{6} \mathrm{~K}$.

The data used here are from the NISAT_S CDS observing sequence that obtains complete NIS spectra over a spatial area $20^{\prime \prime} \times 240^{\prime \prime}$. NISAT_S has been run once per week on quiet Sun regions for the duration of the SOHO mission as part of the CDS synoptic programme. Only studies run up to the (temporary) loss of SOHO in 1998 June are considered here since CDS line profiles were modified following the loss making the weak magnesium lines difficult to measure.

\section{Data preparation}

A list of all runs of the NISAT_S study between the start of science observations (1996 February) and 1998 June 23 was extracted. Through fields in the headers of the data files only those studies positioned within $300^{\prime \prime}$ of Sun centre and not located on an active region were selected. The closest EIT $195^{1}$

\footnotetext{
${ }^{1}$ EIT: extreme Ultraviolet Imaging Telescope, one of the instruments on SOHO. The 195 filter of EIT selects only radiation close to
}

image in time was extracted from the SOHO databases, and the position of the NISAT raster was overplotted allowing the location relative to active regions to be determined. Only rasters lying in clear quiet Sun patches, and not overlying strong bright points were selected. In all, 24 NISAT rasters were chosen. Each were processed with the latest version of the CDS calibration software. The NIS spectra appear tilted on the detector and this was corrected to ensure that each emission line was spatially aligned. Cosmic rays were flagged with the CDS_NEW_SPIKE IDL routine and were not included in the analysis. The data were calibrated in both photon event units and intensity (erg $\mathrm{cm}^{-2} \mathrm{~s}^{-1} \mathrm{sr}^{-1}$ ) units. The errors on the intensity are derived from the photon event values following Thompson (2000). The intensities of some of the stronger lines in the NIS2 wavelength band are affected by the narrow slit burn-in correction (see Sect. 7.7.1 of Lang et al. 2002) that results in line widths (and thus line intensities) falling by up to $10 \%$ over the period 1996-98. The IDL routine GET_WIDTH_CORR in the CDS analysis software corrects for this effect. For the neon lines used in the abundance analysis here, the effects are less than $2 \%$ over the time period considered, and are not accounted for. For the stronger O V $\lambda 629.7$ and Mg X $\lambda 624.9$ lines considered in Sect. 6, however, the intensities can be affected by up to $10 \%$ and have been corrected for.

After the data had been calibrated, the spatial separation into supergranule network and cell centre regions was performed using the $\mathrm{OV} \lambda 629.7$ line. This line is formed at $\log (T / \mathrm{K})=5.4$ and is the strongest transition region line seen by CDS, giving sufficient signal at each spatial pixel to allow a direct separation into network or cell centre regions. Based on the work of Gallagher et al. (1998), 50\% of the quiet Sun is found in network regions and 50\% in cell centres at the temperature of formation of $\mathrm{O} \mathrm{v}$. The spatial pixels in the NISAT rasters were thus divided according to intensity into network and cell centre regions. The intensity value at which the division between network and cell centres occurs for each of the data-sets is shown in Fig. 1. For this and other plots in this paper, time is represented as the number of days since the start of science operations for CDS (1996 February 25). All spatial pixels belonging to the two regions were then summed, and complete NIS spectra extracted.

A Gaussian fitting routine of the author based on the MPFIT curve-fitting procedures of C. B. Markwardt ${ }^{2}$ was used to fit lines in the spectrum. Line intensity errors derived from the fitting procedure were added in quadrature to flux calibration uncertainties, taken as $15 \%$.

\section{Mg and $\mathrm{Ne}$ ions}

Within the two NIS wavebands, CDS observes emission lines from the consecutive ion sequences $\mathrm{Mg} \mathrm{V}-\mathrm{VIII}$ and $\mathrm{Ne}$ IV-VII, for which there is significant temperature overlap (Fig. 2). Thus the $\mathrm{Mg}$ and $\mathrm{Ne}$ ions will be found in the same solar structures and, once the atomic physics parameters are corrected for, the

$195 \AA$ and images are dominated by Fe XII $\lambda 195.1$ emission formed at around 1.5 million $\mathrm{K}$.

${ }^{2}$ http://astrog.physics . wisc . edu/ craigm/idl/idl .html 


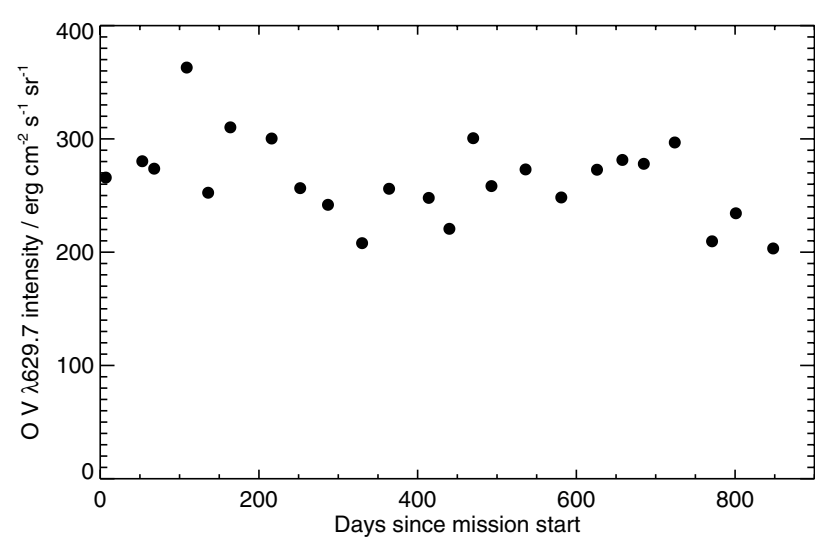

Fig. 1. The O V $\lambda 629.7$ intensity at which the division between network and cell centre regions occurs for each of the 24 data-sets.

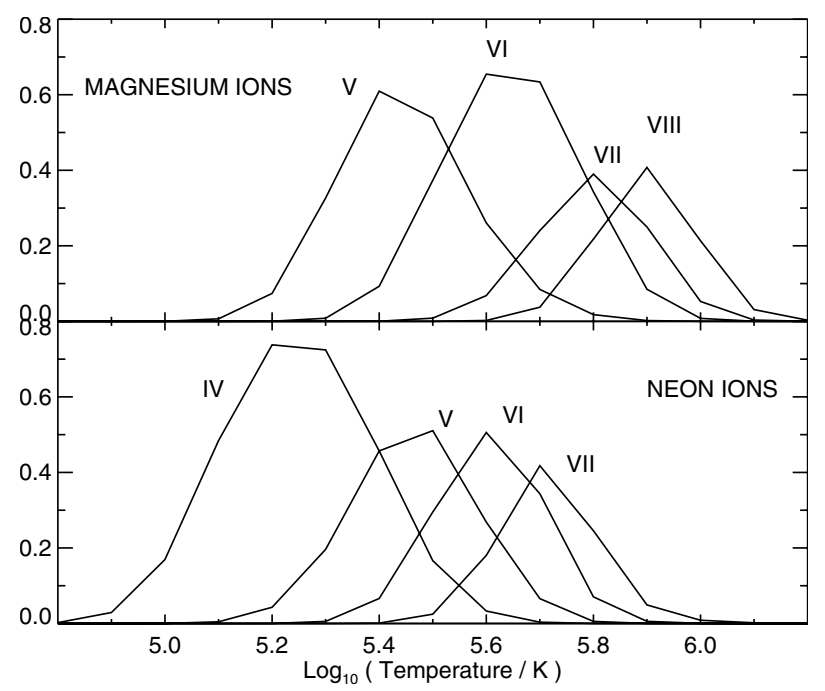

Fig. 2. Ionization fraction curves from Mazzotta et al. (1998) for the magnesium and neon ions.

ratios of the emission lines from the two elements allow a measure of the relative abundance of the two elements. By simulaneously comparing four ions from each element, errors due to atomic data uncertainties in any single ion are avoided.

The transitions used in the present work are listed in Table 1. Whereas most of the lines are unblended and simply fit with a single Gaussian, the $\mathrm{Mg} \mathrm{V}$ and $\mathrm{Mg}$ VII lines are more difficult. The former lies close to the stronger Fe XI $\lambda 352.7$ and Fe XII $\lambda 352.1$ lines and all three were fitted together in a 3-Gaussian fit. In cases where the Mg V line was particularly weak the widths of all three lines were forced to be the same. The Mg VII $\lambda 367.7$ line is partially blended with the stronger Mg IX $\lambda 368.1$ line and the lines were simultaneously fit with two Gaussians forced to have the same width. Note that all lines observed with NIS are dominated by instrumental broadening and thus lines close in wavelength typically have the same width.

Table 1 also lists other emission lines used in this work.
Table 1. Emission lines used in the present analysis. Transitions within $0.4 \AA$ are blended in the CDS spectra. Wavelengths are from v4.2 of the CHIANTI database.

\begin{tabular}{|c|c|c|}
\hline Ion & Transition & $\lambda / \AA$ \\
\hline Mg V & $\begin{array}{l}2 \mathrm{~s}^{2} 2 \mathrm{p}^{4}{ }^{3} \mathrm{P}_{2}-2 \mathrm{~s} 2 \mathrm{p}^{5}{ }^{3} \mathrm{P}_{2} \\
2 \mathrm{~s}^{2} 2 \mathrm{p}^{4}{ }^{3} \mathrm{P}_{1}-2 \mathrm{~s} 2 \mathrm{p}^{5}{ }^{3} \mathrm{P}_{1}\end{array}$ & $\begin{array}{l}353.09 \\
353.30\end{array}$ \\
\hline Mg VI & $\begin{array}{l}2 s^{2} 2 p^{3}{ }^{2} D_{5 / 2}-2 s 2 p^{4}{ }^{2} D_{3 / 2} \\
2 s^{2} 2 p^{3}{ }^{2} D_{3 / 2}-2 s 2 p^{4}{ }^{2} D_{3 / 2} \\
2 s^{2} 2 p^{3}{ }^{2} D_{5 / 2}-2 s 2 p^{4}{ }^{2} D_{5 / 2} \\
2 s^{2} 2 p^{3}{ }^{2} D_{3 / 2}-2 s 2 p^{4}{ }^{2} D_{5 / 2}\end{array}$ & $\begin{array}{l}349.11 \\
349.12 \\
349.16 \\
349.18\end{array}$ \\
\hline Mg VII & $\begin{array}{l}2 \mathrm{~s}^{2} 2 \mathrm{p}^{2}{ }^{3} \mathrm{P}_{2}-2 \mathrm{~s} 2 \mathrm{p}^{3}{ }^{3} \mathrm{P}_{2} \\
2 \mathrm{~s}^{2} 2 \mathrm{p}^{2}{ }^{3} \mathrm{P}_{2}-2 \mathrm{~s} 2 \mathrm{p}^{3}{ }^{3} \mathrm{P}_{1}\end{array}$ & $\begin{array}{l}367.66 \\
367.67\end{array}$ \\
\hline Mg VIII & $2 \mathrm{~s}^{2} 2 \mathrm{p}^{2} \mathrm{P}_{3 / 2}-2 \mathrm{~s} 2 \mathrm{p}^{2}{ }^{2} \mathrm{P}_{3 / 2}$ & 315.02 \\
\hline $\mathrm{Ne}$ IV & $2 s^{2} 2 p^{3}{ }^{4} S_{3 / 2}-2 s 2 p^{4}{ }^{4} P_{5 / 2}$ & 543.89 \\
\hline $\mathrm{Ne} V$ & $2 \mathrm{~s}^{2} 2 \mathrm{p}^{2}{ }^{3} \mathrm{P}_{2}-2 \mathrm{~s} 2 \mathrm{p}^{3}{ }^{3} \mathrm{D}_{1}$ & 572.03 \\
\hline $\mathrm{Ne}$ VI & $\begin{array}{l}2 s^{2} 2 p^{2} \mathrm{P}_{3 / 2}-2 \mathrm{~s} 2 \mathrm{p}^{2}{ }^{2} \mathrm{D}_{3 / 2} \\
2 \mathrm{~s}^{2} 2 \mathrm{p}^{2} \mathrm{P}_{3 / 2}-2 \mathrm{~s} 2 \mathrm{p}^{2}{ }^{2} \mathrm{D}_{5 / 2}\end{array}$ & $\begin{array}{l}562.70 \\
562.80\end{array}$ \\
\hline Ne VII & $\begin{array}{l}2 \mathrm{~s} 2 \mathrm{p}^{3} \mathrm{P}_{1}-2 \mathrm{p}^{2}{ }^{3} \mathrm{P}_{1} \\
2 \mathrm{~s} 2 \mathrm{p}^{3} \mathrm{P}_{2}-2 \mathrm{p}^{2}{ }^{3} \mathrm{P}_{2}\end{array}$ & $\begin{array}{l}561.38 \\
561.73\end{array}$ \\
\hline Si IX & $\begin{array}{l}2 \mathrm{~s}^{2} 2 \mathrm{p}^{2}{ }^{3} \mathrm{P}_{1}-2 \mathrm{~s} 2 \mathrm{p}^{3}{ }^{3} \mathrm{D}_{1} \\
2 \mathrm{~s}^{2} 2 \mathrm{p}^{2}{ }^{3} \mathrm{P}_{1}-2 \mathrm{~s} 2 \mathrm{p}^{3}{ }^{3} \mathrm{D}_{2} \\
2 \mathrm{~s}^{2} 2 \mathrm{p}^{2}{ }^{3} \mathrm{P}_{2}-2 \mathrm{~s} 2 \mathrm{p}^{3}{ }^{3} \mathrm{D}_{1} \\
2 \mathrm{~s}^{2} 2 \mathrm{p}^{2}{ }^{3} \mathrm{P}_{2}-2 \mathrm{~s} 2 \mathrm{p}^{3}{ }^{3} \mathrm{D}_{2} \\
2 \mathrm{~s}^{2} 2 \mathrm{p}^{2}{ }^{3} \mathrm{P}_{2}-2 \mathrm{~s} 2 \mathrm{p}^{3}{ }^{3} \mathrm{D}_{3}\end{array}$ & $\begin{array}{l}344.95 \\
345.12 \\
349.62 \\
349.79 \\
349.86\end{array}$ \\
\hline Mg IX & $2 \mathrm{~s}^{2}{ }^{1} \mathrm{~S}_{0}-2 \mathrm{~s} 2 \mathrm{p}{ }^{1} \mathrm{P}_{1}$ & 368.07 \\
\hline $\operatorname{Mg} X$ & $2 \mathrm{~s}^{2} \mathrm{~S}_{1 / 2}-2 \mathrm{p}{ }^{2} \mathrm{P}_{1 / 2}$ & 624.94 \\
\hline Si XII & $2 s^{2} S_{1 / 2}-2 p{ }^{2} P_{1 / 2}$ & 520.67 \\
\hline
\end{tabular}

\section{5. $\mathrm{Mg} / \mathrm{Ne}$ abundance}

The line fitting procedure resulted in eight intensity values corresponding to the eight magnesium and neon ions. A minimization procedure was then applied to derive the $\mathrm{Mg} / \mathrm{Ne}$ relative abundance using atomic data from the CHIANTI database and a model for the plasma distribution with temperature.

The model discretises the plasma into temperature components distributed at 0.1 dex intervals between $5.0 \leq$ $\log (T / \mathrm{K}) \leq 6.1$. This region covers all temperatures at which the $\mathrm{Mg}$ and $\mathrm{Ne}$ ions are formed (Fig. 2). The theoretical intensity for an emission line is given by

$4 \pi I=0.83 h v \mathrm{Ab}(\mathrm{X}) \sum_{i} n\left(T_{i}, N_{\mathrm{e}}\right) A F\left(T_{i}\right) N_{\mathrm{e}}\left(T_{i}\right) h_{i}$

where $I$ is the intensity, $h v$ the energy for the transition, $\mathrm{Ab}(\mathrm{X})$ the abundance of element $\mathrm{X}, n$ the population of the upper emitting level of the transition (normalised such that the total of all the level populations is set to 1 ), $A$ the radiative decay rate for the transition, $F$ the ionization fraction, $N_{\mathrm{e}}$ the electron number density, and $h_{i}$ the plasma column depth at temperature $T_{i}$. The $n$ and $A$ values are derived from v4.2 of the CHIANTI database (Young et al. 2003) and the $F\left(T_{i}\right)$ values are from Mazzotta et al. (1998). A constant pressure is assumed based on the density derived from the Si IX density diagnostic (Sect. 6). The pressure $\left(=T N_{\mathrm{e}}\right)$ is set to $10^{14.5} \mathrm{~K} \mathrm{~cm}^{-3}$. The effects on the results of assuming constant density instead are discussed below. 


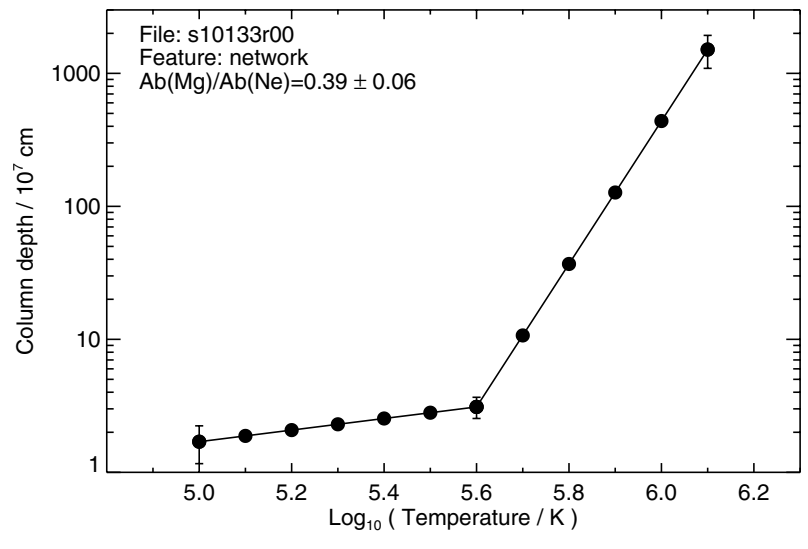

Fig. 3. Column depths $(h)$ derived from the supergranule network spectrum of CDS data-set s10133r00 (1998 January 10). The three values at $\log T=5.0,5.6$ and 6.1 were derived through the minimization procedure, all other values are obtained by linear interpolation from these three values.
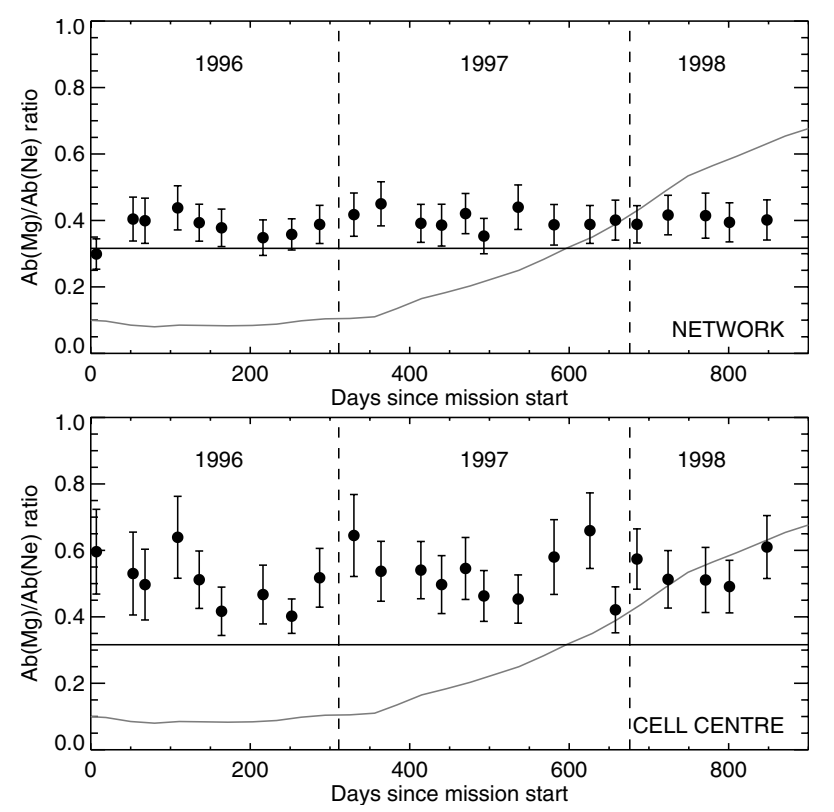

Fig. 4. The derived $\mathrm{Mg} / \mathrm{Ne}$ abundance ratios for the network (upper panel) and cell centre (lower panel) regions as a function of time. The black horiontal line denotes the photospheric $\mathrm{Mg} / \mathrm{Ne}$ abundance ratio. The grey line shows the variation in the sunspot number over the period of observations, from a value of 10 at day 0 to 68 at day 900 .

The $h_{i}$ values are determined through the minimization procedure, although only three of the values enter as free parameters: the values at $\log T=5.0,5.6,6.1$, which we refer to as $h_{0}$, $h_{6}$ and $h_{11}$. The remaining values are determined by linear interpolation in the $\log h-\log T$ plane. An example of the derived $h$ values for one data-set is shown in Fig. 3 .

The MPFIT procedures mentioned previously were used to minimise the difference between the theoretical intensities (derived from Eq. (1)) and observed intensities through the four parameters $\mathrm{Ab}(\mathrm{Mg}) / \mathrm{Ab}(\mathrm{Ne}), h_{0}, h_{6}$ and $h_{11} \cdot \chi^{2}$ values ranged from 0.66 to 3.2 over the different data-sets ${ }^{3}$. The

\footnotetext{
3 The complete set of results from the minimization procedure is given in Table 2 - available online.
}

$\mathrm{Ab}(\mathrm{Mg}) / \mathrm{Ab}(\mathrm{Ne})$ values for the network and cell centre regions are displayed in Fig. 4. The photospheric $\mathrm{Mg} / \mathrm{Ne}$ abundance is taken as $0.316^{4}$ (Grevesse \& Sauval 1998). In both types of region there is no significant trend in time, and the average enhancements over the photospheric $\mathrm{Mg} / \mathrm{Ne}$ abundance are $1.25 \pm 0.10$ (network) and $1.66 \pm 0.23$ (cell centres). The average enhancement of the cell centres over the network is $1.33 \pm 0.21$. Also shown in Fig. 4 is the variation in sunspot number ${ }^{5}$ over the period of the CDS observations. This demonstrates that there is no trend with solar activity. The abundance results are considered to apply over the temperature range $5.3 \leq \log (T / \mathrm{K}) \leq 5.8$ as this is the principle range of overlap of the magnesium and neon ions (Fig. 2), and the division into cell centres and network does not apply at high temperatures.

Assuming a constant density in the minimization procedure produces only small changes in the results. E.g., for a density of $10^{9} \mathrm{~cm}^{-3}$, the network and cell centre abundance ratios are $1.25 \pm 0.11$ and $1.69 \pm 0.25$; while for a density of $10^{10} \mathrm{~cm}^{-3}$ they become $1.20 \pm 0.13$ and $1.67 \pm 0.29$.

In all data-sets the cell centre $\mathrm{Mg} / \mathrm{Ne}$ ratio is greater than the network value. The largest enhancement of cell centre over network is 1.99 while the smallest enhancement is 1.03 .

The average values for the column depths $h_{0}, h_{6}$ and $h_{11}$ are: $64.1,165$ and $72800 \mathrm{~km}$ (cell centres); and 157, 350 and $104000 \mathrm{~km}$ (network). Note that these values assume that neon has its photospheric abundance $(\log \mathrm{Ab}(\mathrm{Ne}) / \mathrm{Ab}(\mathrm{H})=-3.92$, Grevesse \& Sauval 1998) in the transition region.

\section{Other quiet Sun properties}

Complete NIS spectra were extracted in order to study the $\mathrm{Mg} / \mathrm{Ne}$ abundance ratio and other lines in these spectra can be used to determine additional properties of the quiet Sun. Presented below are coronal density and temperature measurements, and line intensities as a function of time.

Two emission lines of Si IX are found at 345.1 and $349.9 \AA$ in the NIS spectra and they are blends of 2 and 3 Si IX transitions (Table 1), respectively. The $\lambda 349.9 / \lambda 345.1$ ratio is density sensitive and the range of sensitivity is particular useful for coronal hole and quiet Sun regions (Fig. 5). Si IX is formed at temperatures of $\approx 1$ million $\mathrm{K}$, and so the separation of cell and network regions has limited physical significance at coronal temperatures. The intensities are thus averaged to yield quiet Sun intensities, and the $\lambda 349.9 / \lambda 345.1$ line ratio is converted to an electron density through software in the CHIANTI database. Figure 6a shows the density values for each of the data-sets. The average value is $2.6_{-0.4}^{+0.5} \times 10^{8} \mathrm{~cm}^{-3}$.

Landi \& Landini (1998) found quiet Sun densities of $5.0_{-2.8}^{+6.2} \times 10^{8}$ and $7.9_{-4.7}^{+11.1} \times 10^{8} \mathrm{~cm}^{-3}$ using SiIX for two quiet Sun regions. We note, however, that the high temperature Si XII $\lambda 520.7$ intensity for these regions is 145 and $254 \mathrm{erg} \mathrm{cm} \mathrm{cm}^{-2} \mathrm{~s}^{-1} \mathrm{sr}^{-1}$, respectively, significantly higher than the values found in the present work (see below and Fig. 6),

\footnotetext{
4 The slightly higher neon abundance quoted by Feldman \& Laming (2000) leads to a photospheric $\mathrm{Mg} / \mathrm{Ne}$ ratio of 0.295 .

5 Obtained from ftp://ftp.ngdc.noaa.gov/STP/SOLAR_DATA/ SUNSPOT_NUMBERS.
} 


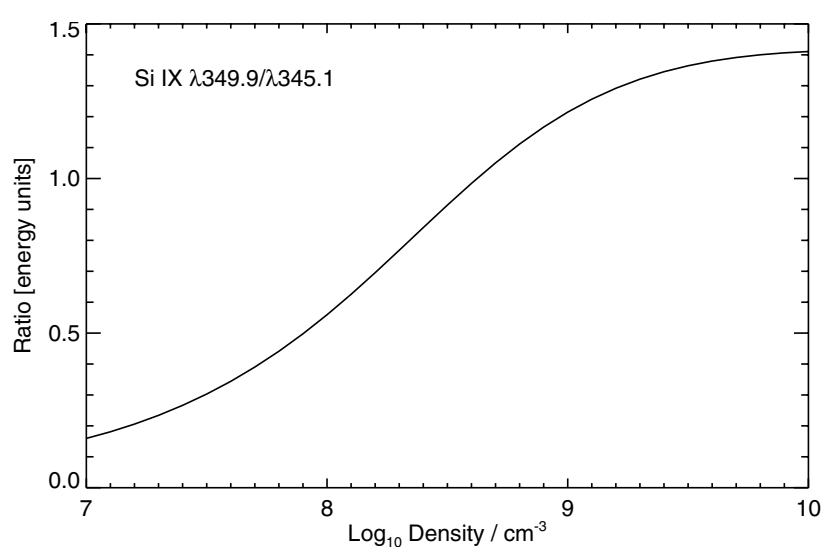

Fig. 5. Variation of the Si IX $\lambda 349.9 / \lambda 345.1$ ratio as a function of density. Calculated with 44.2 of the CHIANTI database at a temperature of $\log (T / \mathrm{K})=6.1$.

indicating that there is some contamination from the nearby active region.

The ratio of the $\mathrm{Mg}$ X $\lambda 624.9$ line to the $\mathrm{Mg}$ IX $\lambda 368.1$ line can be used to yield a temperature estimate for the quiet Sun if the plasma is assumed to be isothermal. Although the solar atmosphere is clearly multithermal, the $\mathrm{Mg} \mathrm{X} / \mathrm{Mg}$ IX ratio is useful since the peak of the emission measure distribution in the quiet Sun is around $\log T=5.9-6.0$ (Del Zanna \& Bromage 1999), close to the temperatures of maximum formation of the two ions. Figure $6 \mathrm{~b}$ shows the temperatures derived from the line ratio. The values are all very similar and there is no trend with time. The average value is $\log (T / \mathrm{K})=5.95 \pm 0.02$.

Figure $6 \mathrm{c}$ shows the variation of three emission lines with time: O V $\lambda 629.7, \operatorname{Mg}$ X $\lambda 624.9$ and Si XII $\lambda 2520.7$. The latter two are coronal lines and so are averaged over the network and cell centre regions. The average intensities in units erg cm $\mathrm{cm}^{-2} \mathrm{~s}^{-1} \mathrm{sr}^{-1}$ are $458 \pm 63,189 \pm 24$ (O V network and cell centre), $29.7 \pm 5.1(\mathrm{Mg} \mathrm{X})$ and $2.1 \pm 1.5$ (Si XII). The very low intensity of Si XII $\lambda 520.7$ (one of the stronger lines in active region spectra) means there is no significant plasma emission above $\log T=6.1$.

\section{Conclusions}

$\mathrm{The} \mathrm{Mg} / \mathrm{Ne}$ abundance ratio in the transition region of the Sun at temperatures $5.3 \leq \log T \leq 5.8$ is enhanced over the value in the photosphere by less than a factor 2 . In supergranule cell centre regions the enhancement factor is $1.66 \pm 0.23$, while for network regions it is $1.25 \pm 0.10$. There is no trend with solar cycle over the 28 month period (1996 Feb. to 1998 Jun.) for which data was analysed.

Simultaneous measurements of the electron density using the density sensitive Si IX $\lambda 349.9 / \lambda 345$.1 line ratio yield an average value of $2.6_{-0.4}^{+0.5} \times 10^{8} \mathrm{~cm}^{-3}$ for the quiet Sun. The $\mathrm{Mg} \mathrm{X}$ 1624.9/Mg IX $\lambda 368.1$ ratio yields an average quiet Sun temperature $\log (T / \mathrm{K})=5.95 \pm 0.02$.

The quiet Sun seen at transition region temperatures is known to be inhomogeneous and schematic models for the magnetic structuring are suggested by Dowdy et al. (1986) and Peter (2001). These indicate that a significant proportion

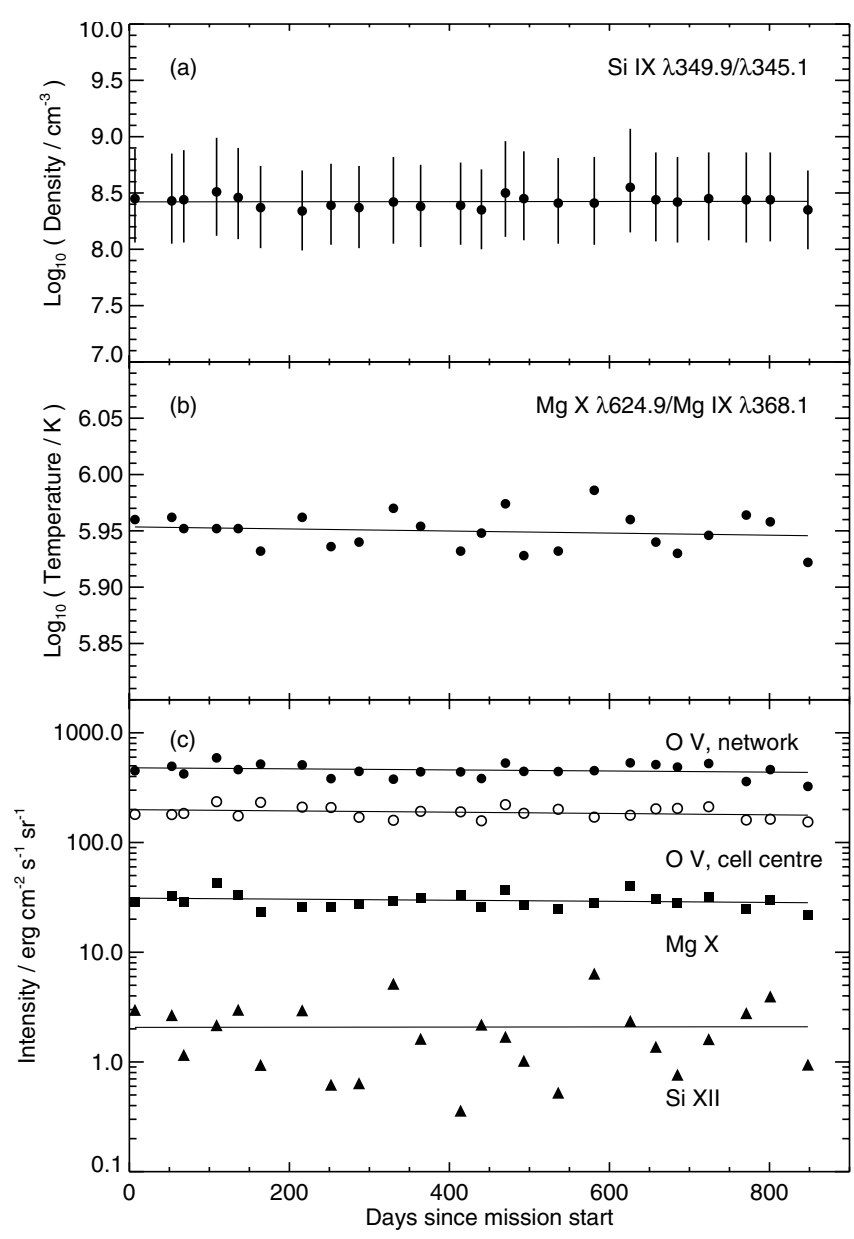

Fig. 6. Variation of spectral properties over the 850 day period considered. a) electron density derived from Si IX $\lambda 349.9 / \lambda 345.1$ diagnostic; b) coronal isothermal temperature derived from $\mathrm{MgX} \lambda 624.9 / \lambda 368.1$ ratio; c) absolute line intensities for three species. In each plot linear fits to the data are shown.

of the emission originates in small closed loops that do not reach coronal temperatures. Direct evidence for these loops is provided by high resolution spectroheliograms obtained by the SUMER instrument on SOHO (Feldman et al. 1999). The abundance results for the quiet Sun presented here show that the slow solar wind $\mathrm{Mg} / \mathrm{Ne}$ relative abundance value is not found in the quiet Sun transition region: Von Steiger et al. (2000) used in situ measurements from the SWICS instrument on Ulysses to determine fast and slow solar wind element abundances for a number of different elements. Their $\mathrm{Mg} / \mathrm{Ne}$ abundance ratio derived for the period 1997 July to 1998 April (which overlaps with the present work) is a factor $4.4 \pm 2.0$ greater than the photospheric value and much higher than the present values. The consequences of this result are twofold. Only a small fraction of the magnetic field lines that thread the quiet Sun transition region can extend out into the solar wind (otherwise the abundance ratios would be the same for both regions). Secondly the closed loops that dominate the transition region emission show close-to photospheric abundances.

Supergranule cell centres contain less closed field and there is a clear enhancement of the $\mathrm{Mg} / \mathrm{Ne}$ ratio over network 
regions as derived from the CDS data. This can be interpreted as a greater contribution of open field line plasma to the total emission from cell centres. The cell centre abundance values are, however, still considerably below the slow solar wind $\mathrm{Mg} / \mathrm{Ne}$ ratio. High spatial resolution, high sensitivity observations of superganule cell centres are required to search for small open-field regions that would be expected to show the high $\mathrm{Mg} / \mathrm{Ne}$ enhancements found in the solar wind.

None of the quantities ( $\mathrm{Mg} / \mathrm{Ne}$ abundance, density, temperature) measured in the present work show a significant trend with time as solar activity increases from its minimum in 1996. In particular, the hottest line in this work (Si XII $\lambda$ 520.7, formed at $\log [T / \mathrm{K}]=6.3$ ) shows an average constant trend with time. This implies that true quiet Sun regions are not affected by the solar cycle. This is consistent with the relatively small changes in quiet Sun magnetic flux during the solar cycle (Hagenaar et al. 2003), which may be indicative of a separate, solar cycle independent, dynamo mechanism that generates the ephemeral regions found in the quiet Sun.

CDS has allowed a detailed study of the FIP effect in the quiet Sun at transition region temperatures due to the simultaneous observation of several ions of magnesium and neon. A similar study for coronal plasma in the quiet sun is not possible with CDS as there are no useful lines of sulphur and argon (coronal lines of neon are found at X-ray wavelengths). However, the EUV Imaging Spectrometer to be flown on SolarB (Culhane et al. 2000) will observe consecutive series of ions from iron and sulphur over 1 to 3 million K. An important project for EIS will be to measure the FIP enhancement at these temperatures in the quiet sun and determine whether it is closer to the solar wind value or the transition region value measured here.

Acknowledgements. The author thanks J. Schmelz, A. Fludra, J. Lang, and the referee, U. Feldman, for useful comments.

\section{References}

Culhane, J. L., Korendyke, C. M., Watanabe, T., \& Doschek, G. A. 2000, SPIE, 4139, 294

Del Zanna, G., \& Bromage, B. J. I. 1999, JGR, 104, 9753

Dowdy, J. F., Rabin, D., \& Moore, R. L. 1986, Sol. Phys., 105, 35

Feldman, U., \& Laming, J. M. 2000, Phys. Scr., 61, 222

Feldman, U., \& Widing, K. G. 1993, ApJ, 414, 381

Feldman, U., Schühle, U., Widing, K. G., \& Laming, J. M. 1998, ApJ, 505, 999

Feldman, U., Widing, K. G., \& Warren, H. P. 1999, ApJ, 522, 1133

Gallagher, P. T., Phillips, K. J. H., Harra-Murnion, L. K., \& Keenan, F. P. 1998, MNRAS, 335, 733

Grevesse, N., Sauval, A. J. 1998, Space Sci. Rev., 85, 161

Hagenaar, H. J., Schrijver, C. J., \& Title, A. M. 2003, ApJ, 584, 1107, 2003

Harrison, R. A., Sawyer, E. C., Carter, M. K., et al. 1995, Sol. Phys., 162,233

Laming, J. M., Drake, J. J., \& Widing, K. G. 1995, ApJ, 443, 416

Landi, E., \& Landini, M. 1998, A\&A, 340, 265

Lang, J., Thompson, W. T., Pike, C. D., Kent, B. J., \& Foley, C. R. 2002, in The Radiometric Calibration of SOHO, ISSI Sci. Report SR-002, ed. A. Pauluhn, M. C. E. Huber, \& R. von Steiger (Noordwijk: ESA Publications Division), 105

MacPherson, K. P., \& Jordan, C. 1999, MNRAS, 308, 510

Malinovsky, L., \& Heroux, M. 1973, ApJ, 181, 1009

Mazzotta, P., Mazzitelli, G., Colafrancesco, S., \& Vittorio, N. 1998, A\&AS, 133, 403

Meyer, J.-P. 1985, ApJS, 57, 173

Peter, H. 2001, A\&A, 374, 1108

Raymond, J. C., Kohl, J. L., Noci, G., et al. 1997, Sol. Phys., 175, 645 Spadaro, D., Zuccarello, F., \& Zappalà, R. A. 1996, A\&A, 308, 970

Thompson, W. T. 2000, CDS Software Note No. 49, ver. 5

Vernazza, J. E., \& Reeves, E. M. 1978, ApJS, 37, 485

von Steiger, R., Schwadron, N. A., Fisk, L. A., et al. 2000, JGR, 105, 27217

Young, P. R., \& Mason, H. E. 1998, Space Sci. Rev., 85, 315

Young, P. R., Del Zanna, G., Landi, E., et al. 2003, ApJS, 144, 135 